\title{
Postural balance in children with Autism Spectrum Disorders
}

\author{
Erika Suenya Gomes Cordeiro ${ }^{1}$ \\ https://orcid.org/0000-0001-6167-0743 \\ Luana Celly Silva Aprígio' \\ https://orcid.org/0000-0002-8636-4678 \\ Cíntia Alves Salgado Azoni ${ }^{1}$ \\ https://orcid.org/0000-0003-2175-9676 \\ Juliana Maria Gazzola ${ }^{1}$ \\ https://orcid.org/0000-0002-9333-1831
}

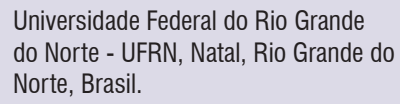

Conflict of interests: Nonexistent

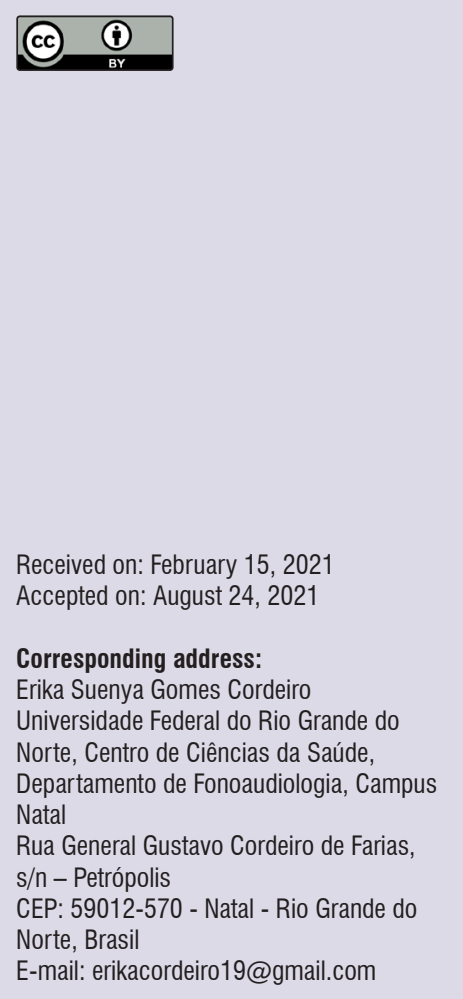

\section{ABSTRACT}

Purpose: to characterize the performance of children with autism spectrum disorders in two postural balance assessment scales.

Methods: an observational cross-sectional study with a descriptive, analytical profile. Seven to 11-year-old children with mild autism spectrum disorder, diagnosed by an interdisciplinary team, according to the DSM-5, were assessed. The protocols used were the Sensory Organization Test and Pediatric Balance Scale.

Results: all the children obtained maximum performance in the Sensory Organization Test. As for the Pediatric Balance Scale, the participants had similar responses in 8 out of its 14 items; in the other 6 , there was a standard deviation.

Conclusion: the participants did not have difficulties performing the Pediatric Balance Scale and Sensory Organization Test, scoring quite close to the maximum value.

Keywords: Autistic Disorder; Vestibular Diseases; Postural Balance; Sensation Disorders; Child; Child Behavior 


\section{INTRODUCTION}

Postural balance (PB) is the person's ability to maintain their body in the desired position, which may be either static or dynamic. PB is made possible by the interaction between the proprioceptive, vestibular, and visual systems ${ }^{1,2}$.

The proprioceptive system informs the body position in relation to surfaces ${ }^{1}$, while the vestibular system informs the head localization in space and transmits information about the body, in relation to the environment, to the central nervous system.

Dizziness and PB problems are present in $5.3 \%$ of children and adolescents in the United States ${ }^{3}$. Children aged 7 years or older can convey better the characteristics of their PB problems ${ }^{4}$.

Everyday movements, such as reaching, grasping, walking, and looking are controlled with the integrated activity of neurocognitive processes, sensory processes, and reflexes. Ongoing movements must be planned, started, guided, monitored, and adjusted to accommodate environmental contingencies ${ }^{5}$.

$\mathrm{PB}$ is one of the requirements necessary to full motor development, daily activity performance, and social interaction adjustment ${ }^{6,7}$. In the case of children, maintaining posture is particularly important, as they are intensely exploring their motor skills and need $\mathrm{PB}$ to reach motor development milestones ${ }^{8}$. During childhood, various deficits, dysfunctions, and disorders may appear, changing the typical course of child development. Childhood may be severely impacted by motor difficulties and other disorders because they further decrease the child's participation in activities with peers, hindering their social interaction and development ${ }^{5}$.

According to the Diagnostic and Statistical Manual of Mental Disorders (DSM-5), neurodevelopmental disorders are deficits that appear during child development and last throughout their life. Although the DSM- 5 describes various disorders, the motor characteristics are not considered diagnostic criteria for most of them ${ }^{9}$. Individuals with these disorders typically have heterogeneous characteristics. Associations between neurodevelopmental disorders are also quite common. It is known that the more comorbidities children have, the worse their performance in various activities is. Children with autism spectrum disorders (ASD) may secondarily have language disorders, attention-deficit/ hyperactivity disorder, intellectual disability, and so forth ${ }^{9,10}$.

ASD is a neurodevelopmental disorder with abnormalities in two groups of characteristics. The first one encompasses language, interaction, and social reciprocity difficulties, while the second one is characterized by repetitive patterns and restricted interests. Sensory abnormalities - which may involve sensory discrimination, perception, and modulation are described in the second group ${ }^{11,12}$. Children with ASD frequently have a hypo- or hyperresponsiveness pattern regarding sensory stimuli ${ }^{9}$.

Though not considered diagnostic criteria for the disorder, motor planning and motor coordination difficulties and atypical gait are also reported ${ }^{9}$. Studies showing sensory discrimination, modulation, and perception difficulties - which are also seen as causes of ASD severity and prognostic - are frequently present in the literature, as well ${ }^{8,13}$. These children's sensory abnormalities explain their aggressiveness, stereotypies, and repetitive patterns $\mathrm{s}^{9,13}$.

People with sensorimotor disorders tend to have difficulties maintaining PB because it characteristically integrates three sensory systems. Therefore, children with ASD are expected to have PB difficulties, as some studies describe ${ }^{14-16}$.

PB regulation is not directed by reflex alone since the upper centers, such as the motor cortex, the basal ganglia, the cerebellum, the vestibular cortex, and the brainstem are involved ${ }^{5}$. Various studies have found that PB is impaired in cases of ASD and that postural abnormalities are predictive of ASD symptomatology ${ }^{17,18}$. More specifically, the increase in postural sway seems to reflect the patients' impaired perception of body movement in relation to their postural limitation boundary, as well as a decreased capacity to separate the distinct ankle and hip movements to align their body when standing ${ }^{19}$.

The more associated difficulties a child has, the worse their prognosis is because integrating sensory information becomes more difficult. As many neural pathways are shared, children with attention deficits, for instance, have greater trouble maintaining their PB while coping with outer stimuli ${ }^{10}$.

Thus, this study aimed to characterize the performance of children presented with ASD on two PB assessment scales.

\section{METHODS}

This is an observational cross-sectional study with a descriptive, analytical profile, approved by the Central Research Ethics Committee of the University Hospital Onofre Lopes, (HUOL, in Portuguese), Brazil, under number 3.232.724. It was carried out at the Center for 
Health Education and Research Anita Garibaldi (CEPE, in Portuguese), located in Macaíba, Rio Grande do Norte, Brazil.

The sample was selected by convenience, analyzing the medical records available at the CEPE. The eligibility criteria were as follows: 7 - to 11-year-old children with mild ASD, diagnosed by an interdisciplinary team according to the DSM-5, with an absence of comorbidities or lesions associated with ASD, not taking medications that might interfere with $\mathrm{PB}$, and without a specific diagnosis of postural abnormality. Children who did not finish the assessment were excluded. All the parents/ guardians signed the informed consent form. Two children were excluded because they did not finish the assessment.

The assessments, in which the parents were present, took place at the CEPE and lasted 45 minutes on average. At first, parents were told how the PB assessment would be conducted, and which items would be assessed. The evaluator asked the child to perform the protocol tasks, giving examples and making it fun. In the end, the test results were explained to the parents.

PB was assessed with two protocols. The first one was the Sensory Organization Test (SOT), whose analysis reveals the sensory interaction between the visual, vestibular, and proprioceptive systems. No recommendations were found regarding the minimum or maximum age to take the test which comprises four sensory conditions described below ${ }^{20}$. The responses to the four tasks are scored from zero to 100 , observing the presence or absence of body sway. Scores from zero to 59 are considered as fall; from 60 to 69 , risk of fall; and from 70 to 100 , normal ${ }^{21}$. The overall balance index, calculating the arithmetic mean of the values found in the four testing conditions, was also measured. The child was asked to maintain each of the sensory conditions in the test for 30 seconds. They were as follows:

- Condition 1: Patient in orthostatic position, eyes open, on an even and steady surface.

- Condition 2: Patient in orthostatic position, eyes closed, on an even and steady surface.

- Condition 3: Patient in orthostatic position, eyes open, on a foam surface.

- Condition 4: Patient in orthostatic position, eyes closed, on a foam surface.

Then, the Brazilian version of the Pediatric Balance Scale (PBS), which has been translated to Portuguese ${ }^{22}$ was applied. This is an adaptation of the Berg Balance
Scale (BBS), whose purpose is to assess the risk of falls in older adults. The pediatric version was developed for schoolchildren aged 5 to 15 years and it assesses the static and dynamic PB with 14 items (Figure 1). Its tasks simulate activities of daily living. In each item, the score ranges from 0 to 4 points, summing a total score of 56 points - the highest score represents the child's full ability to perform all the tasks.

\begin{tabular}{|l|}
\hline Description of the item \\
\hline 1. Sitting to standing \\
\hline 2. Standing to sitting \\
\hline 3. Transfers \\
\hline 4. Standing unsupported \\
\hline 5. Sitting unsupported \\
\hline 6. Standing with eyes closed \\
\hline 7. Standing with feet together \\
\hline 8. Standing with foot in front \\
\hline 9. Standing on one foot \\
\hline 10. Turning 360 degrees \\
\hline 11. Turning to look behind \\
\hline 12. Retrieving object from floor \\
\hline 13. Placing alternate foot on stool \\
\hline 14. Reaching forward with outstretched arm \\
\hline
\end{tabular}

Figure 1. Description of the items in the Pediatric Balance Scale

Data with descriptive statistics, with median, interquartile range, mean, and standard deviation, were analyzed.

\section{RESULTS}

Initially, 14 children aged 7 to 12 years - one girl and 13 boys - participated in this study. All the parents/ guardians were fully informed of the assessment procedures and agreed to participate. However, two children (a boy and a girl) were unwilling to continue after beginning the tests and were excluded from the research. Hence, the final sample comprised 12 boys aged 7 to 11 years (mean 8 and a half years). In general, the participants were receptive to the tasks, which they performed after fun examples and instruction.

The sample children had heterogeneous characteristics but the same diagnosis. Children number 1, 2, 3, and 4 had equine-assisted therapy on the assessment date. The other ones had undergone multidisciplinary assessments and their families had received 
instructions, but they were not having therapy at the time of the assessments.

The PBS results varied between the participants, whereas all the children had maximum performance in the SOT. No child had dizziness or was at risk of fall
- there was a little more sway in condition 4 but with no significant change. Figure 2 shows the children's scores in each PBS item, revealing that most of them had maximum performance in each task.

\begin{tabular}{|c|c|c|c|c|c|c|c|c|c|c|c|c|c|c|c|}
\hline Child & Item & Item & Item & Item & Item & Item & Item & Item & Item & Item & Item & Item & Item & Item & PBSS \\
\hline 1 & 4 & 4 & $\mathbf{3}$ & $\mathbf{4}$ & $\mathbf{5}$ & $\mathbf{6}$ & $\mathbf{7}$ & $\mathbf{8}$ & $\mathbf{9}$ & $\mathbf{1 0}$ & $\mathbf{1 1}$ & $\mathbf{1 2}$ & $\mathbf{1 3}$ & $\mathbf{1 4}$ & \\
\hline 2 & 4 & 4 & 4 & 4 & 4 & 4 & 4 & 4 & 4 & 4 & 4 & 4 & 4 & 4 & 56 \\
\hline 3 & 4 & 4 & 4 & 4 & 4 & 4 & 4 & 4 & 4 & 4 & 4 & 4 & 4 & 4 & 56 \\
\hline 4 & 4 & 4 & 4 & 4 & 4 & 4 & 4 & 4 & 3 & 4 & 4 & 4 & 4 & 4 & 55 \\
\hline 5 & 4 & 4 & 4 & 4 & 4 & 4 & 2 & 0 & 4 & 4 & 4 & 4 & 4 & 4 & 50 \\
\hline 6 & 4 & 4 & 4 & 4 & 4 & 4 & 3 & 4 & 4 & 4 & 4 & 4 & 4 & 4 & 55 \\
\hline 7 & 4 & 4 & 4 & 4 & 4 & 4 & 4 & 4 & 4 & 4 & 4 & 4 & 4 & 2 & 54 \\
\hline 8 & 4 & 4 & 4 & 4 & 4 & 4 & 4 & 4 & 3 & 4 & 4 & 4 & 4 & 3 & 54 \\
\hline 9 & 4 & 4 & 4 & 4 & 4 & 4 & 4 & 3 & 3 & 4 & 4 & 4 & 4 & 4 & 54 \\
\hline 10 & 4 & 4 & 4 & 4 & 4 & 4 & 4 & 3 & 3 & 4 & 3 & 4 & 4 & 4 & 53 \\
\hline 11 & 4 & 4 & 4 & 4 & 4 & 4 & 4 & 4 & 4 & 4 & 4 & 4 & 4 & 3 & 55 \\
\hline 12 & 4 & 4 & 4 & 4 & 4 & 4 & 4 & 4 & 4 & 3 & 4 & 4 & 4 & 4 & 55 \\
\hline
\end{tabular}

Captions:

Item 1: Sitting to standing

Item 2: Standing to sitting

Item 3: Transfers

Item 4: Standing unsupported

Item 5: Sitting unsupported

Item 6: Standing with eyes closed

Item 7: Standing with feet together

Item 8: Standing with foot in front

Item 9: Standing on one foot

Item 10: Turning 360 degrees

Item 11: Turning to look behind

Item 12: Retrieving object from floor

Item 13: Placing alternate foot on stool

Item 14: Reaching forward with outstretched arm

PBSS: Pediatric Balance Scale score

Figure 2. Responses of the participants in the Pediatric Balance Scale

The participants had similar responses in the PBS - only items $7,8,9,10,11$, and 14 had a standard deviation, as shown in Table 1. Other tasks were analyzed, but the values of both the standard deviation and interquartile range were zero, as the participants obtained the same scores in the tasks. 
Table 1. Descriptive analysis of items 7, 8, 9, 10,11, and 14 of the Pediatric Balance Scale in children with autism spectrum disorder

\begin{tabular}{lccc}
\hline & Mean $\mathbf{E S D}$ & Median (IQR 25-75\%) & Reference values \\
\hline PBS & $54.42 \pm 1.67$ & $55(54-55.75)$ & $0-56$ \\
Item 7 & $3.75 \pm 0.62$ & $4(4-4)$ & $0-4$ \\
Item 8 & $3.50 \pm 1.16$ & $4(3.25-4)$ & $0-4$ \\
Item 9 & $3.66 \pm 0.49$ & $4(3-4)$ & $0-4$ \\
Item 10 & $3.91 \pm 0.28$ & $4(4-4)$ & $0-4$ \\
Item 11 & $3.91 \pm 0.28$ & $4(4-4)$ & $0-4$ \\
Item 14 & $3.66 \pm 0.65$ & $4(3.25-4)$ & $0-4$ \\
\hline
\end{tabular}

Captions:

SD: standard deviation; IQR: interquartile range; PBS: Pediatric Balance Scale

Item 7: Standing with feet together

Item 8: Standing with foot in front

Item 9: Standing on one foot

Item 10: Turning 360 degrees

Item 11: Turning to look behind

Item 14: Reaching forward with outstretched arm

\section{DISCUSSION}

The objective of this study was to characterize the performance of children with ASD on two PB assessment scales. In the SOT, the participants obtained maximum values in all four conditions. In the PBS, the final scores varied but with a rather small standard deviation.

The studies by Lim et al. $^{13}$ and Gouleme et al. ${ }^{23}$ compare PB performance between typical children (control group) and children with ASD. The review by Lim et al. ${ }^{13}$ assessed 434 people with ASD and 551 with typical development, aged 3 to 52 years. The study by Gouleme et al. ${ }^{23}$ compared 30 children with typical development with another 30 children with ASD, mean age of $12.1 \pm 2.9$ years. In both studies, the children with ASD had an inferior performance in the PB assessments than those with typical development. The methodological differences may have led to the result in this study, in which there was no abnormal balance, unlike the said articles ${ }^{13,23}$.

Darr et al. (2015) ${ }^{24}$ conducted a PBS psychometric study in 2- to 13-year-old children with typical development. Even though $37 \%$ of those children obtained maximum scores, in general, the final scores varied little ${ }^{24}$ - similar to those found in this study.

The PBS is a validated protocol that proved to be sensitive in PB assessment of children with Down syndrome ${ }^{25}$, chronic encephalopathy ${ }^{26}$, and visual disability $^{27}$. However, no abnormalities were found in the assessment of this study population $-25 \%$ of the children obtained maximum scores and $67 \%$ had slightly lower performance.
In general, the more a child with ASD has associated comorbidities and difficulties, the worse their performance in activities of daily living is - depending on the age at diagnosis and whether there have been adequate interventions. In Radonovich et al. ${ }^{14}$, one reads that children with stereotypies had more PB difficulties. Lim et al..$^{13}$ noticing that children with ASD use the visual system more than the others in the PB tasks, in comparison with typical children. Gouleme et al. ${ }^{23}$ observed that, when exposed to tasks that require more attention, children with ASD have more postural sways on the force platform than expected. In the literature in general, the assessments are made in children with mild autism because of the complexity of the PB tests. Likewise in this study, the children with mild ASD and no specific diagnosis of postural abnormality had adequate $\mathrm{PB}$ in the tools used to assess them.

The children's lowest performance in the PBS occurred in items $7,8,9,10,11$, and 14, which require them, respectively, to stand with feet together, stand with a foot in front, stand on one foot, turn 360 degrees, turn to look behind, and reach forward with outstretched arms.

Tasks that narrow down the base of support, such as items 7,8 , and 9 , increase the sway in children with ASD. The psychometric study ${ }^{24}$ revealed that $82 \%$, $48 \%$, and $48 \%$ obtained maximum performance in these tasks, respectively.

Tasks 10 and 11 assess dynamic PB. One child had a lower score in these two items, which require broad motor coordination and dual-task attention. Miller et al. ${ }^{28}$ and Bucci et al. ${ }^{29}$ observed that the 
PB in children with ASD decreased when they had abnormal broad motor coordination and dual-task attention. In this study, it was seen that children with difficulties in these skills obtained a lower total score. Item 14 is considered an assessment in itself, known as Functional Reach Test. As demonstrated by Duncan et al..$^{30}$, this task assesses PB using body displacement and measuring its maximum capacity. Three children obtained the maximum score in the present study. In the psychometric study, children with ASD in this study had a performance similar to that of typical children.

The SOT assesses the interaction between the vestibular, visual, and proprioceptive systems, which maintains PB. The postural sway in this study was not significant, although the participants were more resistant to the task that required them to stand with a blindfold. All the children were able to stand for the 30 seconds of the test.

In the literature, more difficulties in computerized posturography are verified, which enables the evaluators to digitally observe the interaction between the sensory systems responsible for $\mathrm{PB}^{19,23,29-32}$. These assessments detect even the slightest body sway and the children's need for a larger base of support.

One of the characteristics of ASD in the DSM- 5 is hypo- or hyperresponsiveness to sensory stimuli. Sensory integration difficulties are also described as a requirement in ASD. The SOT may not be sensitive enough to detect slight PB sway in children with mild ASD.

Despite being a protocol often used to assess balance in children, the PBS did not reveal abnormal results in the assessment of this group of children with mild ASD. The tasks seemed too easy for this group at times. Likewise, the SOT without computerized posturography did not have abnormal results. One infers that technological devices, such as force platforms and computerized posturography, can better detect slight sway in children with mild ASD. It must be highlighted that no previous assessment in this study sample verified abnormal PB, which prevents us from extending the result to analyze the instruments' sensitivity.

One of the limiting factors in this study is the sample size. Due to the strict eligibility criteria, many children were not recruited for assessment. The children's medical records were analyzed, in order to select them and recruit the ones that met the inclusion criteria. Children with a more severe ASD, with severe cognitive impairment or some associated disability, were not assessed. Further studies with larger samples that reliably represent children with ASD are recommended. Moreover, the control group should be assessed, and the variables should be better controlled. Hence, the results can be extended.

\section{CONCLUSION}

The participants did not have difficulties performing the PBS and SOT and obtained scores quite close to the maximum. According to the assessment with these scales, the children with ASD did not have PB abnormalities.

\section{REFERENCES}

1. Herdman SJ. Reabilitação vestibular. 2a ed. São Paulo: Manole; 2002.

2. Steindl R, Kunz K, Schrott-Fischer A, Scholtz AW. Effect of age and sex on maturation of sensory systems and balance control. Dev. Med. Child Neurol. 2006;48(6):477-82.

3. Li CM, Hoffman HJ, Ward BK, Cohen HS, Rine RM. Epidemiology of dizziness and balance problems in children in the United States: a population-based study. J. Pediatr. 2016;171(1):240-7.

4. Formigoni LG, Medeiros IRT, Santoro PP, Bittar RSM, Bottino MA. Avaliação clínica das vestibulopatias na infância. BJORL. 1999;65(1):78-82.

5. Stins JF, Emck C, de Vries EM, Doop S, Beek PJ. Attentional and sensory contributions to postural sway in children with autism spectrum disorder. Gait Posture. 2015;42(2):199-203.

6. Mancini M, Horak FB. The relevance of clinical balance assessment tools to differentiate balance deficits. Eur. J. Phys. Rehabil. Med. 2010;46(2):239-48.

7. Pollock AS, Durward BR, Rowe PJ, Paul JP. What is balance? Clin. Rehabil. 2000;14(4):402-6.

8. Doumas M, McKenna R, Murphy B. Postural control deficits in Autism Spectrum Disorder: the role of sensory integration. J. Autism Dev. Disord. 2016;46(3):853-61.

9. American Psychiatric Association. Diagnostic and Statistical Manual of Mental Disorders, quinta edição (DSM-V). Arlington, VA: American Psychiatric Association, 2013.

10. Moseley RL, Pulvermüller F. What can autism teach us about the role of sensorimotor systems in higher cognition? New clues from studies on language, 
action semantics, and abstract emotional concept processing. Cortex. 2018;100:149-90.

11. Blanche El, Reinoso G, Chang MC, Bodison S. Proprioceptive processing difficulties among children with autism spectrum disorders and developmental disabilities. Am. J. Occup. Ther. 2012;66(5):621-4.

12. Fournier KA, Hass CJ, Naik SK, Lodha N, Cauraugh $\mathrm{JH}$. Motor coordination in autism spectrum disorders: a synthesis and meta-analysis. J. Autism Dev. Disord. 2010;40(10):1227-40.

13. Lim YH, Partridge K, Girdler S, Morris SL. Standing postural control in individuals with Autism Spectrum Disorder: systematic review and meta-analysis. J. Autism Dev. Disord. 2017;47(7):2238-53.

14. Radonovich KJ, Fournier KA, Hass CJ. Relationship between postural control and restricted, repetitive behaviors in autism spectrum disorders. Front Integr Neurosci. 2013;7:28.

15. Ghanouni P, Memari AH, Gharibzadeh S, Eghlidi J, Moshayedi P. Effect of social stimuli on postural responses in individuals with Autism Spectrum Disorder. J. Autism Dev. Disord. 2017;47(5):1305-13.

16. Rosario MG, López L, Méndez M, Ababneh AF, Gonzalez-Sola M. Proprioception and vestibular alterations affect postural control in children with mild autism: a pilot study. F1000Research. 2018;7:305.

17. MacDonald M, Lord C, Ulrich DA. The relationship of motor skills and social communicative skills in school-aged children with autism spectrum disorder. Adapt Phys Activ Q. 2013;30(3):271-82.

18. Mickle KJ, Munro BJ, Steele JR. Gender and age affect balance performance in primary school-aged children. J Sci Med Sport. 2011;14(3):243-8.

19. Wang Z, Hallac RR, Conroy KC, White SP, Kane $A A$, Collinsworth AL et al. Postural orientation and equilibrium processes associated with increased postural sway in autism spectrum disorder (ASD). J Neurodev Disord. 2016;8:43.

20. Shumway-Cook A, Horak FB. Assessing the influence of sensory interaction of balance. Suggestion from the field. Phys Ther. 1986;66(10):1548-50.

21. Oda DTM, Ganança CF. Computerized dynamic posturography in the assessment of body balance in individuals with vestibular dysfunction. Audiol., Commun. Res. 2015;20(2):89-95.
22. Ries LGK, Michaelsen SM, Soares PSA, Monteiro VC, Allegretti KMG. Cross-cultural adaptation and reliability analysis of the Brazilian version of Pediatric Balance Scale (PBS). Braz. J. Phys. Ther. 2012;16(3):205-15.

23. Gouleme N, Scheid I, Peyre H, Seassau M, Maruani $\mathrm{A}$, Clarke $\mathrm{J}$ et al. Postural control and emotion in children with Autism Spectrum Disorders. Transl Neurosci. 2017;8:158-66.

24. Darr N, Franjoine MR, Campbell SK, Smith E. Psychometric properties of the Pediatric Balance Scale using Rasch analysis. Pediatr Phys Ther. 2015;27(4):337-48.

25. Leite JC, Neves JCJ, Vitor LGV, Fujisawa DS. Postural control in children with Down syndrome: evaluation of functional balance and mobility. Rev. Bras. Educ. Espec. 2018;24(2):173-82.

26. Yi SH, Hwang JH, Kim SJ, Kwon JY. Validity of pediatric balance scales in children with spastic cerebral palsy. Neuropediatrics. 2012;43(6):307-13.

27. Żyłka JL, Lache U, Rutkowska I. Functional Balance Assessment with Pediatric Balance Scale in girls with visual impairment. Pediatr Phys Ther. 2013;25(4):460-6.

28. Miller HL, Caçola PM, Sherrod GM, Patterson RM, Bugnariu NL. Children with Autism Spectrum Disorder, Developmental Coordination Disorder, and typical development differ in characteristics of dynamic postural control: a preliminary study. Gait post. 2019;67(1):9-11.

29. Bucci MP, Contenjean CDY, Kaye K. The effect of performing a dual task on postural control in children with autism. ISRN Neurosci. 2013;2013:796174.

30. Duncan PW, Weiner D, Chandler J, Studenski S. Functional reach: predictive validity in a sample of elderly male veterans. J Gerontol A Biol Sci Med Sci. 1992;47(3):93-8.

31. Greffou S, Bertone A, Hahler EM, Hanssens JM, Motron L, Faubert J. Postural hypo-reactivity in autism is contingent on development and visual environment: a fully immersive virtual reality study. J. Autism Dev. Disord. 2012;42(6):961-70.

32. Molloy CA, Dietrich KN, Bhattacharya A. Postural stability in children with autism spectrum disorder. J. Autism Dev. Disord. 2003;33(6):643-52. 